\title{
Maternal and neonatal outcome of spontaneous versus induced labour at term
}

\section{Shrestha D', Bajracharya J², Shrestha NS ${ }^{3}$}

'Dipty Shrestha, Lecturer; ${ }^{2} J u n u$ Bajracharya, Assistant Professor; ${ }^{3}$ Nira Singh Shrestha, Associate Professor; Department of Obstetrics and Gynaecology, Kathmandu Medical College Teaching Hospital, Kathmandu, Nepal.

\begin{abstract}
Background: Induction of labour today has become the most common obstetric intervention. It is increasing worldwide but there are still controversies regarding its benefits.

Objective: To compare the maternal and neonatal outcomes of elective induction of labour with spontaneous onset of labour in pregnant women from 40 to 42 weeks of gestation at Kathmandu Medical College and Teaching Hospital (KMCTH). Methods: This was a comparative hospital based study that was conducted at KMCTH from $15^{\text {th }}$ April 2013 to $15^{\text {th }}$ April 2014. Three hundred women with singleton pregnancies from 40 to 42 weeks of gestation without any risk factors were enrolled and among these 150 women were selected for induction of labour by purposive sampling. Among these, those with Bishops score less than six were induced with prostaglandin $E_{2}$ which was administered intracervically followed by intravenous infusion of oxytocin in an escalating dose. Those with Bishops score more than six were directly induced with intravenous oxytocin in an escalating dose. Another 150 women were allowed to go into spontaneous onset of labour. The outcome that were looked upon were mode of delivery, rate of caesarean section, maternal morbidities which included post partum haemorrhage (PPH), haematoma, third and fourth degree perineal tears, neonatal intensive care unit (NICU) admission and also neonatal morbidities and mortality.

Results: Among 150 pregnant women undergoing induction of labour, 82 (54.6\%) had normal delivery, 10 (6.6\%) had instrumental delivery and $58(38.8 \%)$ had caesarean section whereas among another 150 pregnant women with spontaneous onset of labour 57 (38\%) had normal delivery, 9 (6\%) had instrumental delivery and 84 (56\%) had caesarean section. Regarding the neonatal morbidities, NICU admissions were more in those with spontaneous onset of labour than induced labour; 20 (13.3\%) and 14 (9.3\%) respectively. The commonest indication for neonatal intensive care unit admission was meconium stained liquor.

Conclusion: Elective induction of labour is associated with lesser rates of caesarean section and does reduce the NICU admissions when compared with spontaneous onset of labour.
\end{abstract}

Key words: Caesarean Section, Induced labour, Neonatal Intensive Care Unit, Oxytocin, Prostaglandin, Spontaneous labour

\section{INTRODUCTION}

T he approach to the problem during labour and delivery has substantially changed in the last decade.

Among the various obstetric interventions induction of labour is the most common intervention. Induction of labour is carried out in over $20 \%$ of pregnancies in

Address for correspondence

Dr. Dipty Shrestha

Lecturer

Department of Obstetrics and Gynaecology

Kathmandu medical College Teaching Hospital

E-mail address: diptyshrestha@hotmail.com developed countries ${ }^{1}$. It is indicated when interrupting the pregnancy is thought to be advantageous for the mother or baby and is often carried out for prolonged pregnancies (>41 weeks gestation), where it has been shown to decrease perinatal mortality ${ }^{2}$. Induction of labor before 42 completed weeks of gestation increased steadily in the United States between 1990 and 20103,4.

It is necessary to outweigh the risks and benefits of induction of labour. While indications of labour induction are not definitive, the most common indication for induction is post term pregnancy, which is known to carry increased risk of perinatal mortality, meconium aspiration, and intrauterine infection for the neonate as 
well as increased risk of perineal trauma, labour dystocia, and cesarean delivery for the mother ${ }^{5}$.

Induction of labour at term with no other medical complication decreases both maternal and neonatal morbidities but it may be associated with certain adverse outcomes. One known adverse outcome of induced labor is an increased rate of caesarean section, clearly demonstrated in nulliparous patients ${ }^{6-8}$.

There are many controversies regarding the advantages of induction of labour at term. There are no strict indications or benefit of elective induction of labour when compared to spontaneous onset of labour. So this study was conducted at Kathmandu Medical College Teaching Hospital (KMCTH) with the objectives to compare the maternal and neonatal outcomes of elective induction of labour with spontaneous onset of labour in terms of mode of delivery, maternal and neonatal morbidities and Neonatal Intensive Care Unit (NICU) admission.

\section{METHODS}

This study was a comparative hospital based study conducted at Kathmandu Medical College and Teaching Hospital. The study period was of one year from $15^{\text {th }}$ April 2013 to $15^{\text {th }}$ April 2014. The study population included 300 obstetric cases. The inclusion criteria included in the study were women with singleton, live pregnancies with cephalic presentation and gestational age between 40 to 42 weeks with reactive non stress test (NST) and without any risk factor like intrauterine growth restrictions, oligohydramnious, severe pre-eclampsia, rheumatic heart disease. The exclusion criteria were previous caesarean section, preterm prelabour rupture of membrane, malpresentation and multiple pregnancies.

Performa was developed which included all the relevant points focusing on the objectives of the study. Among these 150 cases fulfilling the inclusion criteria were selected by purposive sampling for induction of labour and were induced with prostaglandin $E_{2}$ (dinoprostone) and oxytocin depending on the Bishop's score after taking the informed consent. Those with Bishop's score less than six were induced with two doses of intracervical prostaglandin $E_{2}$ at an interval of eight hours followed by intravenous infusion of oxytocin in an escalating dose the next day. Those with Bishops Score more than six were directly induced with intravenous oxytocin in an escalating dose. Another 150 cases were allowed to go for spontaneous onset of labour with close fetal well being was assessment by daily fetal kick count charting and biweekly NST. Cases were managed according to hospital protocol if it developed any sign of fetal and or maternal compromise during the waiting period for spontaneous onset of labour.

This study looked upon the mode of delivery, rate of caesarean section, maternal morbidities such as third and fourth degree perineal tear, haematoma, PPH and also NICU admission and perinatal mortality.

All the data was entered in SPSS 20.0 and analysed. Data was presented in frequencies and percentages .The statistical test applied was chi square test and $p$ value < 0.05 was considered statistically significant

Chi-square test was applied to measure relationship between mode of delivery and types of labour (spontaneous and induced).

Large sample test ( $\mathrm{z}$ test) was applied to compare proportion difference in caesarean section among the two groups (induced labour vs. spontaneous labour)

\section{RESULT}

Among the total 300 cases most of them were in the age group of 19-29 years $261(87 \%)$ and $192(64 \%)$ of them were primigravida as shown in Table 1.

Median age for spontaneous and induced labour is 25.5 and 25.7 respectively.

Among the total 150 cases who were induced, $54.6 \%$ had normal delivery, $6.6 \%$ had instrumental delivery and $38.8 \%$ had caesarean section and among the another 150 cases who underwent spontaneous onset of labour $38 \%$ had normal delivery, $6 \%$ had instrumental delivery and $56 \%$ had caesarean section as shown in Table 2 .

Table 1: Distribution of Participants According to the Age Group and Parity

\begin{tabular}{lccc} 
Variables & $\begin{array}{c}\text { Spontaneous } \\
\text { Labour } \\
(\mathbf{n = 1 5 0 )}\end{array}$ & $\begin{array}{c}\text { Induced } \\
\text { Labour } \\
(\mathbf{n = 1 5 0 )}\end{array}$ & Total \\
\hline $\begin{array}{l}\text { Age distribution } \\
\text { <19years }\end{array}$ & 0 & 6 & 6 \\
19-24 years & 61 & 61 & 122 \\
25-29 years & 89 & 50 & 139 \\
>= 30 years & 0 & 33 & 33 \\
Parity & & & \\
Primigravida & 99 & 93 & 192 \\
Multigravida & 51 & 57 & 108 \\
\hline
\end{tabular}


Table 2: Distribution of modes of delivery among study groups

\begin{tabular}{lccc}
\hline Modes of delivery & Spontaneous labour & Induced Labour & P-Value \\
\hline Normal delivery & $57(38 \%)$ & $82(54.6 \%)$ & $10(6.6 \%)$ \\
\hline Instrumental Delivery & $9(6 \%)$ & $58(38.8 \%)$ & 0.009 \\
Caesarean Section & $84(56 \%)$ & $\mathbf{1 5 0}$ & \\
Total & $\mathbf{1 5 0}$ &
\end{tabular}

P value measured by Chi-square test applied

Table 3: Distribution According to Reasons for NICU Admission

\begin{tabular}{|c|c|c|c|c|}
\hline \multirow{2}{*}{$\begin{array}{l}\text { Reasons for NICU } \\
\text { admissions }\end{array}$} & \multicolumn{2}{|c|}{ Spontaneous onset of labour $(n=20)$} & \multicolumn{2}{|c|}{ Elective induction labour $(n=14)$} \\
\hline & ND (6\%) & Caesarean Section (7.3\%) & ND (4.6\%) & Caesarean Section (4.6\%) \\
\hline Big baby & 2 & 2 & 1 & 2 \\
\hline Birth Asphyxia & 2 & 1 & 2 & 1 \\
\hline Meconium Aspiration & 4 & 5 & 2 & 2 \\
\hline Fetal Distress & 1 & 3 & 2 & 2 \\
\hline Total & 9 & 11 & 7 & 7 \\
\hline
\end{tabular}

No statistical difference in the proportion of NICU admission between the spontaneous and the induced labour groups. (20/150 $=0.13$ vs $14 / 150=0.093$, P-value $=0.27$ )

Caesarean section rate was found to be significantly higher among the spontaneous labour group.

There were no such maternal morbidities like third and fourth degree perineal tear, Post Partum haemorrhage and haematoma.

In the group with spontaneous onset of labour 20 neonates were admitted in the NICU. Similarly in the group where mothers underwent elective induction of labour, 14 neonates were admitted to the NICU as shown in Table 3.

\section{DISCUSSION}

Induction of labour at term pregnancy today is increasing widely worldwide but before going for induction of labour, it is necessary to identify its pros and cons. At KMCTH the obstetric admission has increased tremendously in the last two years.

The indication for induction of labour are various; may or may not be complicated with medical conditions. In a study by Barthelemy Tandu-Umba post term pregnancies covered only $14.6 \%{ }^{12}$. In a study by John D.Yeast, Angela Jones and Mark Poskins, postdated pregnancy was found to be the most common indication ${ }^{13}$.

Another major objective of this study was to see the rate of the caesarean section that accounted for $38.8 \%$ in those with elective induction of labour and $56 \%$ in those with spontaneous onset of labour. A study similar to this by Boulvain et al where elective induction of labour was compared with expectant management also showed lower rate of caesarean section in those with induced labour ${ }^{14}$. In another study by Aaron B and colleagues, expectant management of pregnancy was associated with a $22 \%$ increase in caesarean delivery ${ }^{15}$. But in a study by Dunne and Colleagues, elective induction was associated with an increased risks of caesarean section when compared with spontaneous onset of labour ${ }^{16}$. A study by Barthelemy revealed $66.9 \%$ of vaginal delivery and $29.6 \%$ of caesarean section ${ }^{12}$. No maternal morbidities like third and fourth degree tear, $\mathrm{PPH}$, vaginal haematoma were identified in this study though few studies have identified such maternal morbidities.

Most of the women included in this study were between $19-29$ years of age that is $87 \%$ and maximum of them were primigravida about $64 \%$.

This study also looked upon another important outcome that is neonatal outcome. NICU admission was more in those with spontaneous onset of labour. Not much difference was found in those with elective induction of labour and spontaneous onset of labour regarding the adverse neonatal outcome. Among the NICU admissions 9.3\% were in those with elective induction of labour and $13.3 \%$ in those with spontaneous onset of labour and among these most were admitted for meconium stained liquor. A study by Rosallie $\mathrm{M}$ et al confirmed that there is a continuum of risk for both maternal and infant leading upto 41 weeks gestation with the lowest risk of maternal 
and neonatal morbidity and mortality occurring with birth at a gestational age between $37-39$ weeks ${ }^{17}$.

\section{LIMITATION}

A large scale study is required to come to a definite conclusion which is lacking in our study.

\section{CONCLUSION}

Elective induction of labour at term is associated with lower rates of caesarean section and does reduce the NICU admissions to some extent when compared with spontaneous onset of labour.

\section{REFERENCES}

1. Mealing $\mathrm{NM}$, Roberts $\mathrm{CL}$, Ford $\mathrm{JB}$, Simpson $\mathrm{JM}$, Morris JM. Trends in induction of labour, 1998-2007: a population-based study. Aust NZ J Obstet Gynaecol. 2009;49:599-605.

2. Gulmezoglu AM, Crowther CA, Middleton P. Induction of labour for improving birth outcomes for women at or beyond term. Cochrane Database Syst Rev 2006;(4):CD004945.

3. Chauhan SP, Ananth CV. Induction of labor in the United States: a critical appraisal of appropriateness and reducibility. Semin Perinatol 2012;36:336-43.

4. Murthy K, Grobman WA, Lee TA, Holl JL. Trends in induction of labor at early-term gestation. Am J Obstet Gynecol.2011;204:435.e1-6.

5. American College of Obstetricians and Gynecologists Committee on Practice BulletinsObstetrics. ACOG practice bulletin no. 55 (replaces practice pattern no. 6, October 1997): management of postterm pregnancy. Obstet Gynecol. 2004; 104:639-46.

6. Dublin S, Lydon-Rochelle M, Kaplan RC, et al. Maternal and neonatal outcomes after induction of labor without an identified indication. Am J Obstet Gynecol 2000 Oct ; 183:986-94

7. Dunne C, Da SO, Schmidt G, Natale R. Outcomes of elective labour induction and elective caesarean section in low-risk pregnancies between 37 and 41 weeks' gestation. J Obstet Gynaecol Can. 2009;31:1124-30.
8. Glantz JC. Elective induction vs. spontaneous labor associations and outcomes. J Reprod Med. 2005;50:235-40.

9. Barthélémy Tandu-Umba, Robert Laala Tshibangu, Andy Mbangama Muela. Maternal and perinatal outcomes of induction of labor at term in the university clinics of Kinshasa, DR Congo. Open Journal of Obstetrics and Gynecology. 2013; 3:154157.

10. John D. Yeast, Angela Jones and Mary Poskin. Induction of labor and the relationship to cesarean delivery: A review of 7001 consecutive inductions. Am J Obstet Gynecol. 1999 Mar; 180:628-33.

11. Boulvain M, Marcoux S, Bureau M, Fortier M, Fraser W.Risks of induction of labour in uncomplicated term pregnancies. Paediatr Perinat Epidemiol. 2001;15:131-8.

12. Caughey $A B$, Sundaram V, Kaimal AJ, Gienger $A$, Cheng YW, McDonald KM et al. Systematic Review: Elective Induction of Labor Versus Expectant Management of Pregnancy. Ann Intern Med. 2009;151:252-263.

13. Dunne C, Da Silva O, Schmidt G, Natale R. Outcomes of elective labour induction and elective caesarean section in low-risk pregnancies between 37 and 41 weeks' gestation. J Obstet Gynaecol Can. 2009;31:1124-30.

14. Grivell RM, Reilly AJ, Oakey H, Chan A, Dodd JM. Maternal and neonatal outcomes following induction of labor: a cohort study. Acta Obstet Gynecol Scand. 2012; 91:198-203. 